\title{
Repair of articular cartilage defects in rabbits through tissue-engineered cartilage constructed with chitosan hydrogel and chondrocytes*
}

\author{
Ming $\mathrm{ZHAO}^{1,3}$, Zhu CHEN ${ }^{1}$, Kang LIU ${ }^{1}$, Yu-qing WAN ${ }^{2}$, Xu-dong $\mathrm{LI}^{2}$, \\ Xu-wei $\mathrm{LUO}^{1}$, Yi-guang $\mathrm{BAI}^{1}$, Ze-long YANG ${ }^{1}$, Gang $\mathrm{FENG}^{\dagger 1}$ \\ ('Institute of Tissue Engineering and Stem Cells, Nanchong Central Hospital and the Second Clinical Institute of \\ North Sichuan Medical University, Nanchong 637000, China) \\ $\left({ }^{2}\right.$ Department of Orthopaedic Surgery, University of Virginia School of Medicine, Charlottesville 22908, USA) \\ $\left({ }^{3}\right.$ Department of Orthopaedic Surgery, Pixian People Hospital, Pixian 611730, China) \\ †E-mail: fenggangncch@163.com
}

Received Feb. 4, 2015; Revision accepted June 9, 2015; Crosschecked Oct. 20, 2015

\begin{abstract}
Objective: In our previous work, we prepared a type of chitosan hydrogel with excellent biocompatibility. In this study, tissue-engineered cartilage constructed with this chitosan hydrogel and costal chondrocytes was used to repair the articular cartilage defects. Methods: Chitosan hydrogels were prepared with a crosslinker formed by combining 1,6-diisocyanatohexane and polyethylene glycol. Chitosan hydrogel scaffold was seeded with rabbit chondrocytes that had been cultured for one week in vitro to form the preliminary tissue-engineered cartilage. This preliminary tissue-engineered cartilage was then transplanted into the defective rabbit articular cartilage. There were three treatment groups: the experimental group received preliminary tissue-engineered cartilage; the blank group received pure chitosan hydrogels; and, the control group had received no implantation. The knee joints were harvested at predetermined time. The repaired cartilage was analyzed through gross morphology, histologically and immunohistochemically. The repairs were scored according to the international cartilage repair society (ICRS) standard. Results: The gross morphology results suggested that the defects were repaired completely in the experimental group after twelve weeks. The regenerated tissue connected closely with subchondral bone and the boundary with normal tissue was fuzzy. The cartilage lacuna in the regenerated tissue was similar to normal cartilage lacuna. The results of ICRS gross and histological grading showed that there were significant differences among the three groups $(P<0.05)$. Conclusions: Chondrocytes implanted in the scaffold can adhere, proliferate, and secrete extracellular matrix. The novel tissue-engineered cartilage constructed in our research can completely repair the structure of damaged articular cartilage.
\end{abstract}

Key words: Articular cartilage, Chitosan hydrogel, Repair, Tissue engineering doi: $10.1631 /$ jzus.B1500036

Document code: A

CLC number: R318.08

\footnotetext{
Corresponding author

* Project supported by the National Natural Science Foundation of China (Nos. 81171472, 81201407, and 81071270), the Innovation Team Project of Sichuan Provincial Education Department (No. 13TD0030), the Major Transformation Cultivation Project of Sichuan Provincial Education Department (No. 15CZ0021), and the Science and Technology Project of Nanchong City (No. 14A0021), China (1) ORCID: Ming ZHAO, http://orcid.org/0000-0003-4505-948X; Gang FENG, http://orcid.org/0000-0002-2814-4319

(C) Zhejiang University and Springer-Verlag Berlin Heidelberg 2015
}

\section{Introduction}

Articular cartilage is a load-bearing in the structural organization of the normal joint and an essential part of joint motion. However, due to the lack of blood supply, it has limited capacity for regeneration once it is injured, and is usually repaired with fibrous tissue which does not have the durability 
and special mechanics of normal hyaline cartilage (Messent et al., 2007). Therefore, various methods have been designed to promote the cartilage repair. In 1994, transplantation of autologous chondrocytes was reported to facilitate cartilage repair (Brittberg et al., 1994), but this method has many disadvantages, including chondrocyte dedifferentiation during expansion in vitro, easy suspension of injected cells, and impaction of injected cells in cell sap. Other methods included microfracture (Steadman et al., 2002), abrasion and drilling (Dzioba, 1988), and cartilage scraping (Holmes, 2004), but there were still many inherent problems. The repairs were functionally inadequate in the long term and increased the risk of further damage. Based on these reasons, the repair methods of damaged articular cartilage are limited and the traditional treatments are not ideal. As a result, the treatment of cartilage defects has remained a severe challenge in the orthopedic clinic.

Tissue-engineered cartilage may provide a promising approach for therapy. Tissue-engineered cartilage is constructed by seeding cells on materials, which can simulate the survival environment of chondrocytes. At present, synthetic and natural materials are the main components of tissue-engineered scaffold. Synthetic materials have been used widely in biomedicine because of their excellent plasticity, mechanical properties, and biodegradability. However, their poor biocompatibility has limited their further application. Natural materials, such as chitosan (Kuo and Lin, 2006), collagen (de Franceschi et al., 2005), and atelocollagen gel (Ochi et al., 2002), have been receiving increasing attention. Among these natural materials, chitosan bioactive scaffold is ideal for cartilage because of its excellent biocompatibility and antibacterial properties. It can also improve woundhealing in the connective tissues of several species (Kim et al., 2008). Furthermore, some characteristics of chitosan are similar to the extracellular matrix of articular cartilage, such as hyaluronic acid and glycosaminoglycan (GAG) (Francis Suh and Matthew, 2000). Alginate-chitosan microcapsules have been used to encapsulate mouse osteoblastic MC3T3-E1 cells (Qiao et al., 2014), and the results showed that the activity of cells in the alginate-chitosan microcapsules exceeded that of the alginate microcapsule group, which proved that chitosan has excellent biocompatibility. Nettles et al. (2002) demonstrated that cartilage cells can adhere for a long time to chitosan scaffolds, which keeps their morphological character- istics. These studies indicated that chitosan is a desirable scaffold for the repair of cartilage defects.

In this study, we prepared chitosan hydrogels with natural ethylene glycol chitosan. The crosslink agent was prepared by reacting polyethylene glycol (PEG) and 1,6-diisocyanatohexane (HDI). It was then reacted with the amidine and the hydroxyl group of ethylene glycol chitosan to form the network structure (Chen et al., 2014). The preparation and the properties of this chitosan hydrogel have been described in the study of Chen et al. (2014). This kind of chitosan hydrogel has excellent biocompatibility and mechanical properties because small amounts of crosslinkers form a massive covalent bond.

We used this gel to construct tissue-engineered cartilage with costal chondrocytes. Then the tissueengineered cartilage was implanted into defective rabbit articular cartilage. At a predetermined time, the joints were harvested and the repair was evaluated through gross morphology and histological and immunohistochemical analyses. The group implanted with chitosan hydrogel was the blank group, and the group without any treatment was the control group. We have discovered no report on the same topic so far.

\section{Materials and methods}

\subsection{Construction of tissue-engineered cartilage in vitro}

Japanese white rabbits (male or female, average weight $(0.5 \pm 0.1) \mathrm{kg})$ were provided by the Experimental Animals Center of North Sichuan Medical College, Nanchong, China. All animals were anesthetized with pentobarbital sodium $(30 \mathrm{mg} / \mathrm{kg})$. The costal cartilage was harvested from the rabbits and processed in the lab under aseptic conditions. Then the costal cartilage was minced into pieces of about $1 \mathrm{~mm}^{3}$ and incubated by $0.25 \%(2.5 \mathrm{~g} / \mathrm{L})$ collagenase (Sigma, USA) in a water bath overnight at $37^{\circ} \mathrm{C}$. The digested cartilage tissue was centrifuged at $1500 \mathrm{r} / \mathrm{min}$ for $5 \mathrm{~min}$, and then was transplanted to a T75 flask and cultured in 4-(2-hydroxyethyl)-1-piperazineethanesulfonic acid (HEPES)-buffered Dulbecco's modified Eagle's medium (H-DMEM) and 20\% fetal bovine serum (FBS; Hyclone, USA) with $5 \% \mathrm{CO}_{2}$ in an incubator at $37^{\circ} \mathrm{C}$. The first medium change was performed after $24 \mathrm{~h}$, and then the medium was changed every three days. Chondrocytes were passaged and expanded. Chondrocytes in the passage 2 were collected to serve 
as seeding cells. All manipulations were performed under sterile conditions.

The chitosan hydrogel was prepared as published previously (Chen et al., 2014). In this study, discs of $1 \mathrm{~mm}$ diameter were cut from lyophilized chitosan hydrogel scaffold and sterilized by ultraviolet (UV) irradiation in a polystyrene petri dish overnight. The scaffolds were then placed in a 24-well culture plate (one scaffold per well). Chondrocyte suspension $\left(1 \times 10^{7}\right.$ cells $\left./ \mathrm{ml}, 100 \mu \mathrm{l}\right)$ was deposited in the scaffold for $4 \mathrm{~h}$ and then $2 \mathrm{ml}$ of DMEM medium containing $20 \%$ FBS was added. The scaffold with chondrocytes was incubated at $37^{\circ} \mathrm{C}$ with $5 \% \mathrm{CO}_{2}$ for one week before being implanted into the defective cartilage. The chondrocytes-hydrogel was frozen at $-20{ }^{\circ} \mathrm{C}$ and $5-\mu \mathrm{m}$ sections were prepared for normal hematoxylin and eosin ( $\mathrm{H} \& \mathrm{E})$ staining and immunochemical staining.

\subsection{Construction of animal model}

The experimental protocol was approved by the Experimental Animals Center of North Sichuan Medical College. Fifty-four healthy Japanese white rabbits (male or female, average weight $(3.0 \pm 0.2) \mathrm{kg}$ ) were provided by the Experimental Animals Center of North Sichuan Medical College. All rabbits were kept in a controlled environment with access to food and water. The rabbits were anesthetized by injection of $30 \mathrm{mg} / \mathrm{kg}$ of pentobarbital sodium. The knee joints were opened via a lateral parapatellar skin incision, and the patella was dislocated laterally. The knee was flexed $90^{\circ}$ to expose the non-weight-bearing area of the medial femoral condyle. A full-thickness cartilage defect ( $4 \mathrm{~mm}$ in diameter, $3 \mathrm{~mm}$ in depth) was created in the center of the interchondylar groove through use of a circular stainless steel punch (Fig. 1), as reported by Wakitani et al. (1994). The joint capsule was carefully sutured. The soft tissue was returned to its original site. All rabbits were given an injection of $80000 \mathrm{U}$ of penicillin after surgery for $3 \mathrm{~d}$ and allowed free movement post-operation. The 54 model rabbits were divided into two groups according to gender, then they were randomly assigned to three groups as follows: the experimental group (Group 1, $n=18)$ which received tissue-engineered cartilage; the blank group (Group 2, $n=18$ ) in which defects were filled only with the chitosan hydrogel; and the control group (Group 3, $n=18$ ) which received no treatment. At four, eight, and twelve weeks after implantation, the rabbits in each group were killed by injecting overdose pentobarbital sodium, and then the knee joints were harvested for further research.

\subsection{Gross morphology}

The entire knee of each rabbit was dissected and the distal part of each femur was extirpated. The samples from each group were photographed and examined for evaluation according to the International Cartilage Repair Society (ICRS) gross morphology assessment scale for cartilage repair, as shown in Table 1 (Brittberg and Peterson, 1998; Peterson et al., 2000).

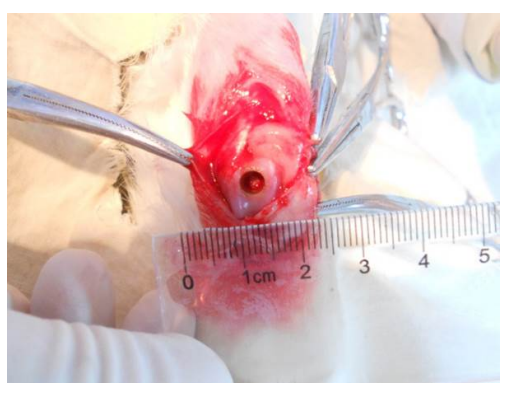

Fig. 1 Construction of animal model

Table 1 International Cartilage Repair Society (ICRS) gross morphology assessment scale

\begin{tabular}{lc}
\hline \multicolumn{1}{c}{ Cartilage repair assessment ICRS } & Point \\
\hline Degree of defect repair & 4 \\
In level with surrounding cartilage & 3 \\
75\% repair of defect depth & 2 \\
$50 \%$ repair of defect depth & 1 \\
25\% repair of defect depth & 0 \\
0\% repair of defect depth & \\
Integration to border zone & 4 \\
Complete integration with surrounding cartilage & 3 \\
Demarcating border $<1$ mm & 2 \\
3/4 of graft integrated, 1/4 with a notable border & 2 \\
$\quad>1$ mm width & \\
1/2 of graft integrated with surrounding carti- & 1 \\
$\quad$ lage, $1 / 2$ with a notable border $>1$ mm & \\
From no contact to $1 / 4$ of graft integrated with & 0 \\
$\quad$ surrounding cartilage & \\
Macroscopic appearance & \\
Intact smooth surface & 4 \\
Fibrillated surface & 3 \\
Small, scattered fissures, or cracks & 2 \\
Several, small, or few but large fissures & 1 \\
Total degeneration of grafted area & 0 \\
Overall repair assessment & \\
Grade I: normal & 12 \\
Grade II: nearly normal & $11-8$ \\
Grade III: abnormal & $7-4$ \\
Grade IV: severely abnormal & $3-1$ \\
\hline B & \\
\hline
\end{tabular}

Brittberg and Peterson, 1998; Peterson et al., 2000 


\subsection{Histological and immunohistochemical analyses}

After gross examination, the lower ends of the femurs were fixed in $4 \%$ buffered formalin for $1 \mathrm{~d}$ and demineralized in decalcifying solution for at least $10 \mathrm{~d}$. Then they were embedded in paraffin and cut into $5-\mu \mathrm{m}$ sections. Sections were stained with $\mathrm{H} \& \mathrm{E}$, safranin- $\mathrm{O} /$ fast, and immunohistochemical staining. Histological observation was performed using a light microscope (Olympus, Japan). The repaired tissue was graded by three observers using the ICRS visual histological assessment scale for cartilage repair, as shown in Table 2 (Mainil-Varlet et al., 2003).

Table 2 International Cartilage Repair Society (ICRS) visual histological assessment scale for cartilage repair

\begin{tabular}{|c|c|}
\hline Feature & Point \\
\hline \multicolumn{2}{|l|}{ I. Surface } \\
\hline Smooth/continuous & 3 \\
\hline Discontinuities/irregularities & 0 \\
\hline \multicolumn{2}{|l|}{ II. Matrix } \\
\hline Hyaline & 3 \\
\hline Mixture: hyaline/fibrocartilage & 2 \\
\hline Fibrocartilage & 1 \\
\hline Fibrous tissue & 0 \\
\hline \multicolumn{2}{|l|}{ III. Cell distribution } \\
\hline Columnar & 3 \\
\hline Mixed/columnar clusters & 2 \\
\hline Clusters & 1 \\
\hline Individual cells/disorganized & 0 \\
\hline \multicolumn{2}{|l|}{ IV. Cell population viability } \\
\hline Predominantly viable & 3 \\
\hline Partially viable & 1 \\
\hline$<10 \%$ viable & 0 \\
\hline \multicolumn{2}{|l|}{ V. Subchondral bone } \\
\hline Normal & 3 \\
\hline Increased remodeling & 2 \\
\hline Bone necrosis/granulation tissue & 1 \\
\hline Detached/fracture/callus at base & 0 \\
\hline \multicolumn{2}{|c|}{ VI. Cartilage mineralization (calcified cartilage) } \\
\hline Normal & 3 \\
\hline Abnormal/inappropriate location & 0 \\
\hline
\end{tabular}

Mainil-Varlet et al., 2003

The expression of collagen II in the regenerated tissue was analyzed by immunohistological staining using a collagen staining kit (Chondrex, USA). The sections were dewaxed in xylene and hydrated through graded alcohol. Then the sections was treated with $2 \%(0.02 \mathrm{~g} / \mathrm{ml})$ bovine testicular hyaluronidase for $30 \mathrm{~min}$ at $25^{\circ} \mathrm{C}$, followed by incubation with blocking buffer for $30 \mathrm{~min}$. The sections were incubated with collagen II monoclonal antibodies (diluted at a volume ratio of $1: 250$ ) overnight at $4{ }^{\circ} \mathrm{C}$. After being washed three times by phosphate-buffered saline (PBS), streptavidin peroxidase (diluted at a volume ratio of 1:200) was added and incubated for $1 \mathrm{~h}$ at $25^{\circ} \mathrm{C}$. Staining was developed in diaminobenzidine (DAB) solution for $20-30$ min at $25^{\circ} \mathrm{C}$.

\subsection{Statistical analysis}

All samples in the three groups were stained and the representative results were listed. The ICRS scores were assess by three people to get the average scores. All data are expressed as the mean \pm standard deviation (SD). To assess differences in macroscopic scoring data and histological scoring data, the statistical analysis was performed with the Student's $t$-test (comparisons between two groups). The significant difference was set at $P<0.01$.

\section{Results}

\subsection{Formulation of chitosan hydrogel and recon- struction of tissue-engineered cartilage in vitro}

The chitosan hydrogel appeared in a columnar lucid shape (Fig. 2a). The chondrocyte-hydrogel was cultured in H-DMEM containing 20\% FBS for one week. $\mathrm{H} \& \mathrm{E}$ staining showed that cells grow confluently on the scaffold and immunohistochemical staining showed that chondrocyte on the scaffold can secrete specific extracellular matrix (Figs. 2c-2d). These results suggested that the tissue-engineered cartilage is already under construction.

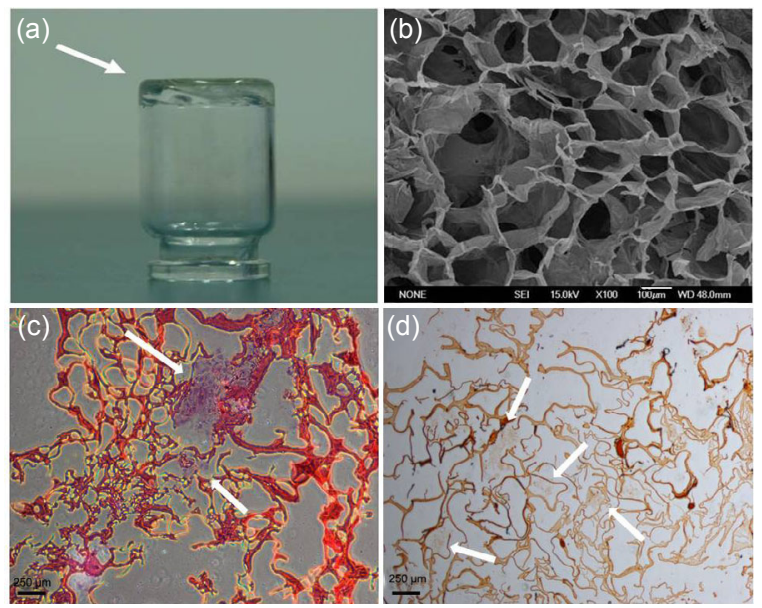

Fig. 2 Morphology of chitosan hydrogel and histology of chondrocytes cultured in chitosan hydrogels

(a) Gross morphology; (b) Scanning electron microscopy, scale bar $=100 \mu \mathrm{m}$; (c) H \& E staining, scale bar $=250 \mu \mathrm{m}$; (d) Immunohistochemical staining, scale bar $=250 \mu \mathrm{m}$ 


\subsection{Macroscopic observations of the articular car- tilage repair twelve weeks post-operation}

All rabbits in the experimental group, with the exception of two, survived the follow-up period of four, eight, and twelve weeks without wound infection, limited range of motion, or synovitis in the operated knees. Generally speaking, the repairs in the experimental group were remarkably better than those in the other two groups. No regenerated tissue existed in the defects in the control group at four weeks. The defect was the same as the model (Fig. 3a). In contrast, some regenerated tissue could be observed at four weeks in the other two groups, although the boundary was still notable between the defective tissue and the normal tissue (Figs. 3d and 3g). The hyaline-like regenerated tissues were found in the experimental and blank groups, but this tissue was far greater in the experimental group. At eight weeks, there was slight tissue repair in the control group, but most defects were still concave, irregular, or sometimes empty in the middle (Fig. 3b). By this time, the defects in the experimental group were repaired with hyaline-like tissue and the joint surface was relatively smooth, although the boundary still existed (Fig. 3h). The defect in the blank group was filled with regenerated tissue, but a gap still existed between the regenerated and normal tissue (Fig. 3e). The defect in the control group was filled with glossy regenerated tissue, but the depression was apparent until twelve weeks later (Fig. 3c). The defect in the experimental group was repaired with hyaline-like regenerative tissue that was

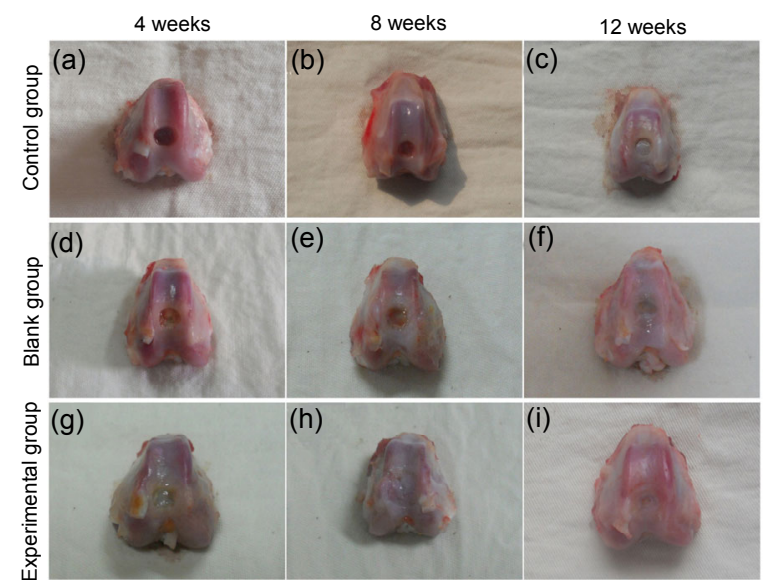

Fig. 3 Gross morphology of repaired cartilage of the control group ( $(, b, c)$, the blank group $(d, e, f)$, and the experimental group $(\mathrm{g}, \mathrm{h}, \mathrm{i})$ at $4(\mathrm{a}, \mathrm{d}, \mathrm{g}), 8(\mathrm{~b}, \mathrm{e}, \mathrm{h})$, and $12(c, f, i)$ weeks similar to normal tissue. The joint surface was smooth and consistent (Fig. 3i). The repair of the defect in the blank group was the same except that the color and transparency of the tissue and the smoothness of the joint surface were worse than those in the experimental group (Fig. 3f).

According to the ICRS scores from macroscopic observations, the average scores in the experimental group were higher than those in the other two groups at every time. The statistical differences between the three groups were significant $(P<0.05$; Fig. 4a).
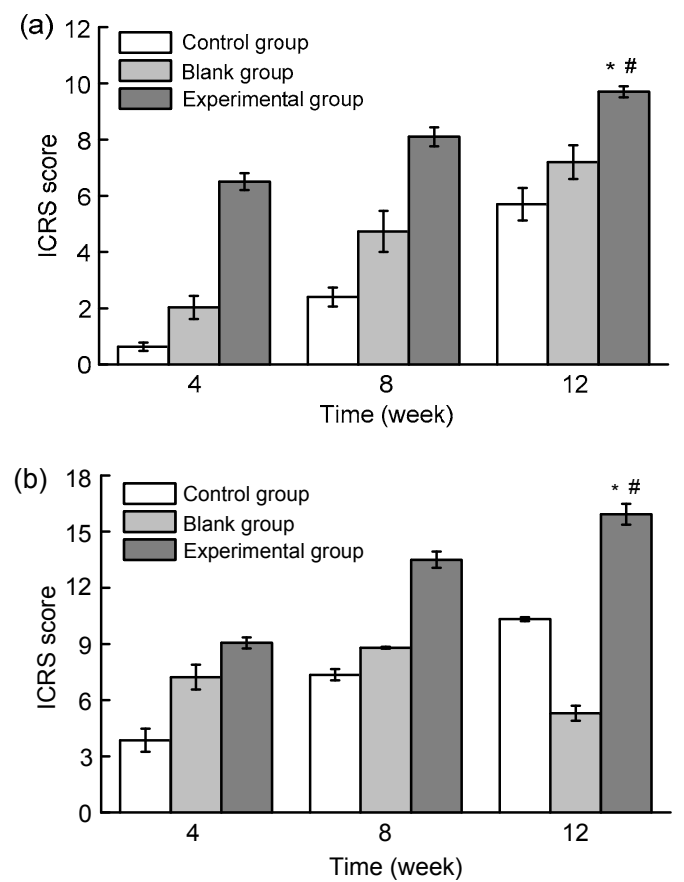

Fig. 4 International Cartilage Repair Society (ICRS) macroscopic assessment scale

(a) Gross morphology assessment; (b) Histological and immunohistochemical assessment. Values are expressed as mean \pm SD $(n=3)$. ${ }^{*} P<0.05$, vs. the control group; ${ }^{\#} P<0.05$, vs. the experimental group ( 8 weeks)

\subsection{Histological and immunohistochemical evalua- tions of the articular cartilage repair twelve weeks post-operation}

In order to further analyze the degree of the repair, all the tissues were embedded in paraffin and cut into sections for histological and immunohistochemical evaluations. The results confirmed that preliminary tissue-engineered cartilage can promote the repair of articular cartilage defects. The defects were obviously repaired in the experimental group by the 
fourth week after implantation. A thin cartilage surface containing some chondrocyte-like cells could be observed, but the surface was lower than the surface of normal articular cartilage (Fig. 5g). Safranin-O staining revealed the existence of proteoglycan in the relatively thin repaired tissue (Fig. 6). Also, immunohistochemical staining indicated that the new tissue can secrete extracellular matrix (Fig. 7). Results from staining showed that at the eighth week, the repair was better in the experimental group (Fig. 5h).
Safranin-O staining revealed obvious cartilage lacuna structure (Fig. 6h) and the secretion of proteoglycan and collagen was also revealed by immunohistochemical staining (Figs. $6 \mathrm{~h}$ and $7 \mathrm{~h}$ ). The defects were repaired completely on gross morphology after twelve weeks. The regenerated tissue closely connected with subchondral bone, and the boundary with normal tissue was fuzzy. The cartilage lacuna in the regenerated tissue was similar to normal cartilage lacuna. It was confirmed that the regenerated tissue was mainly
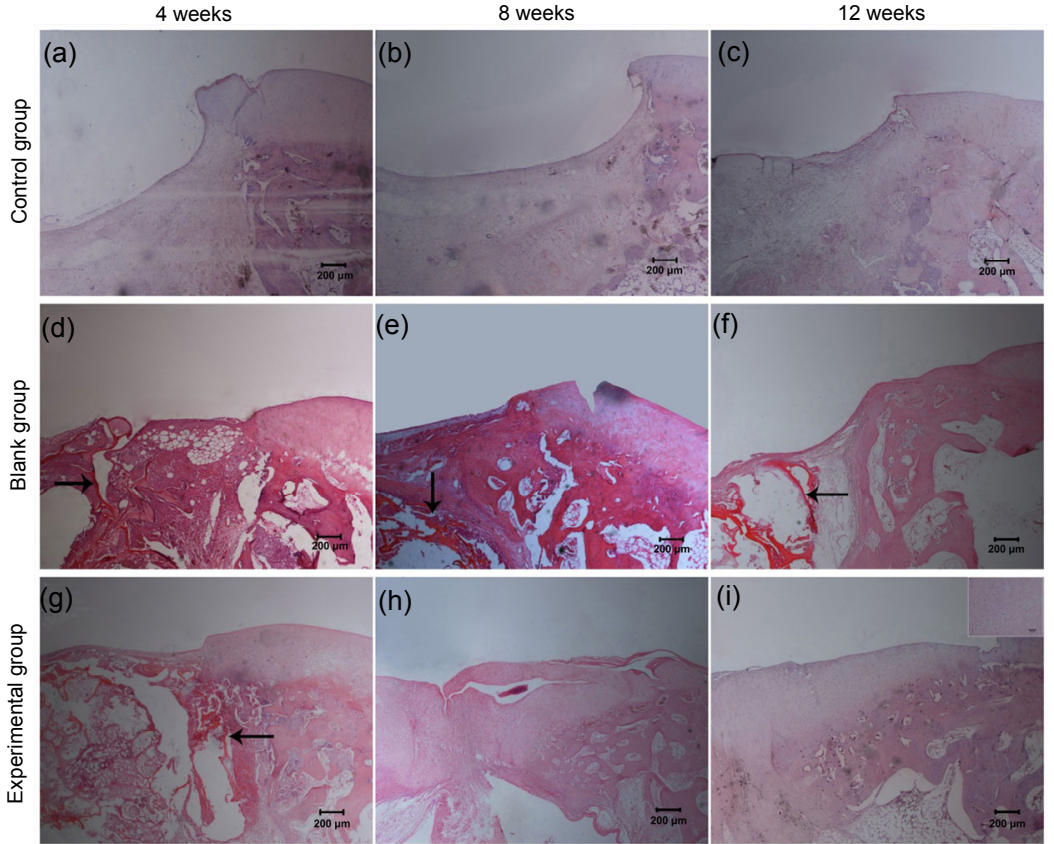

(h)
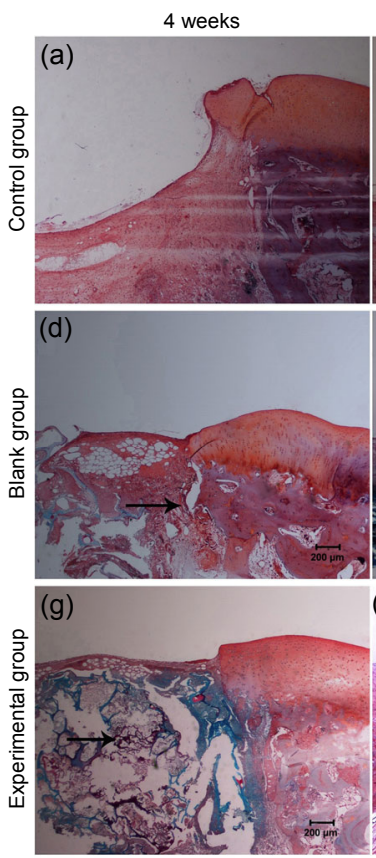

(e)

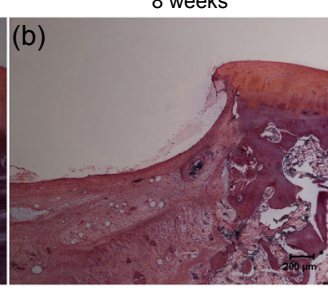

e) (f)

(f)

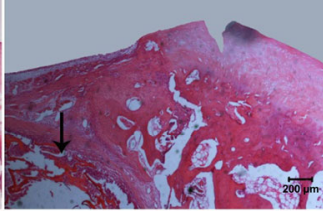

(i)

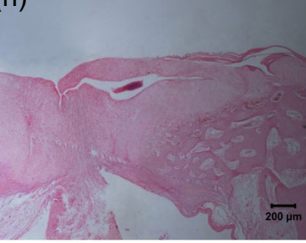

8 weeks

(e)

(h)

(i)

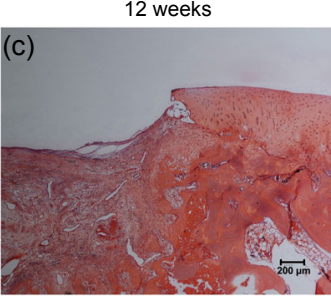

(f)

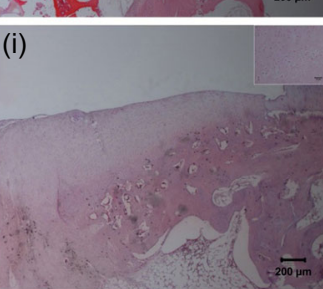

f)
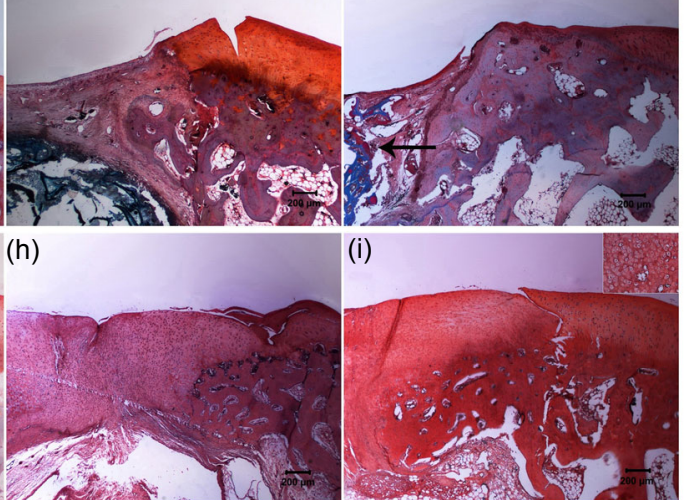

Fig. 5 H \& E staining of repaired cartilage of the control group (a, $b, c)$, the blank group (d, e, f), and the experimental group $(\mathrm{g}, \mathrm{h}$, i) at $4(\mathrm{a}, \mathrm{d}, \mathrm{g}), 8(\mathrm{~b}, \mathrm{e}, \mathrm{h})$, and 12 (c, f, i) weeks

Scale bar $=200 \mu \mathrm{m}$. Arrows show the chitosan scaffold

Fig. 6 Safranin-O staining of repaired cartilage of the control group (a, b, c), the blank group $(d, e, f)$, and the experimental group (g, h, i) at 4 (a, d, g), 8 (b, e, h), and 12 (c, f, i) weeks

Scale bar $=200 \mu \mathrm{m}$. Arrows show the chitosan scaffold 

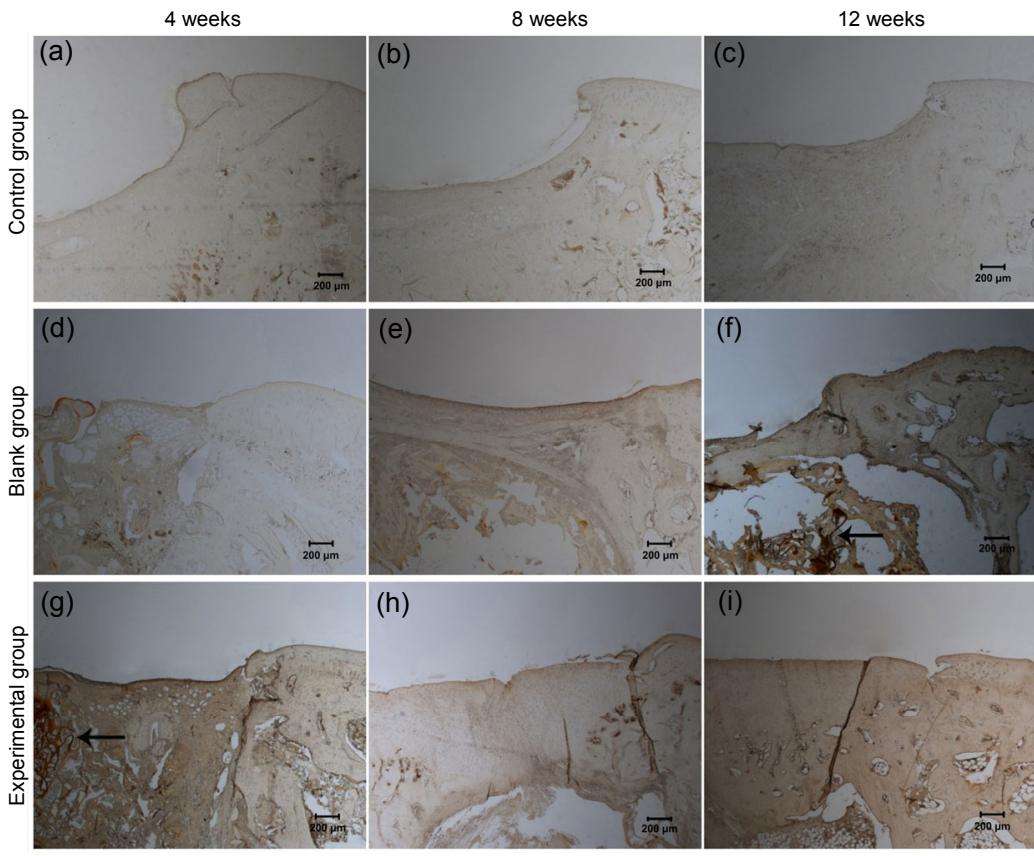

(h) (f)

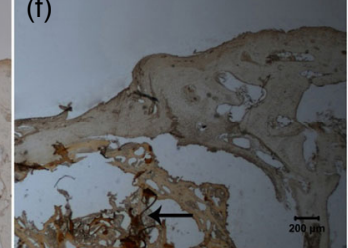

(i)

\section{(1)} .

Fig. 7 Immunochemical staining of collagen II of the control group $(a, b, c)$, the blank group $(d, e, f)$, and the experimental group $(\mathrm{g}, \mathrm{h}, \mathrm{i})$ at $4(\mathrm{a}, \mathrm{d}, \mathrm{g}), 8(\mathrm{~b}, \mathrm{e}$, h), and $12(c, f, i)$ weeks

Scale bar $=200 \mu \mathrm{m}$. Arrows show the chitosan scaffold

hyaline-like cartilage. Compared with the experimental group, the defects in the blank group were only repaired partially with time. From the results of $\mathrm{H} \& \mathrm{E}$ staining and safranin-O staining, we could conclude that the regenerative tissue in the blank group was mainly fibrous tissue with a small amount of hyaline-like tissue (Figs. 5d and 6d). The repair tissue could also secrete extracellular matrix, such as proteoglycan and collagen II (Figs. 6 and 7). The repair of the defect in the control group was the worst. Twelve weeks after surgery, the joint surface of the defects was still concave and was filled with fibrous tissue and a little chondrocyte-like tissue (Figs. $5 \mathrm{c}$ and 6c). In addition, the secretion of collagen II was the worst in the three groups (Fig. 7).

Histological analysis was evaluated according to ICRS scores. As shown in Fig. 4b, the average score was consistent with the results of histological staining and immunohistochemical staining, with the exception of the score for the blank group at twelve weeks. This score was lower than those of the other two groups at the same time.

\section{Discussion}

Articular cartilage damaged by disease or trauma has a limited capacity for regeneration. The selfrepair of articular cartilage differs based on levels of blood supply, innervation, and the degree of damage. However, generally speaking, damage is repaired by chondrocytes in the subchondral bone plate or mesenchymal stem cells, inflammatory cells and cytokines in the bone marrow. Ultimately, damage is repaired by fibrous cartilage (Roach et al., 2007; Richter, 2009). From the histological staining results in the control group, we observed that the defects were filled with fibrous cartilage (Figs. 5-7), which is consistent with articular cartilage self-repair. Fibrous cartilage easily degenerates and eventually develops into osteoarthritis (Peterson et al., 2010), so the clinical treatment of defective cartilage is still a troublesome problem (Wakitani et al., 1998).

Tissue engineering represents a new approach for articular cartilage repair. This technique reconstructed living tissue by associating cells with threedimensional biomaterials which provided a scaffold for cells to proliferate under physiological conditions (Iwasa et al., 2009). The application of new materials in tissue-engineered scaffolds has received much more and more attention (Fong et al., 2012; Mallick and Cox, 2013). More and more studies have confirmed that scaffold material simulating the living microenvironment could better simulate the physiological characteristics of organ (Perka et al., 2000; Lee and Mooney, 2001; Jin et al., 2009). As we know, collagen and proteoglycans were the main articular cartilage extracellular matrixes. Proteoglycan is 
formed by protein covalent with sulfated GAG, and chitosan is one of the polysaccharide natural materials, which contains many amino groups (Gentili and Cancedda, 2009). The composition of chitosan is similar to that of proteoglycan, so it can simulate the articular cartilage extracellular matrix to some extent and could promote the formation of cartilage tissue (Liu et al., 2010). In our previous work (Chen et al., 2014), we prepared a novel chitosan hydrogel, which contained only $1 \%$ (volume ratio) chemical crosslinker, minimizing the cytotoxicity caused by the crosslinker and preserving chitosan's natural properties, and also reported the relative experiment results. In this study, we constructed tissue-engineered cartilage with chitosan hydrogel in vitro, and tried to repair articular cartilage defects in vivo. The pure chitosan hydrogel scaffold was set as the blank group. The $\mathrm{H} \& \mathrm{E}$ staining results in both the experimental and the blank groups at the three time points showed no obviously inflammatory cells, such as neutrophilic granulocytes, lymphocytes, or granulocytes, indicating that chitosan hydrogel has excellent biocompatibility (Fig. 5). The gross morphology, histological and immunohistochemical results all indicated that the repairs in the two groups were notably better than that of the control group (Figs. 3, 5-7). Hyaline-like cartilage was observed at the twelfth week, especially in the experimental group (Fig. 6i). The ICRS assessment showed that the repair results in the experimental group were the best. From these results, we conclude that articular cartilage defects can be repaired by tissue-engineered cartilage constructed with chitosan hydrogel prepared in our lab. It should be noted that the defect in the blank group was partially repaired (Figs. 3d-3f, 5d-5f, and 6d-6f), which indicates that, because of its biocompatibility and antibacterial properties, pure chitosan hydrogel can also promote cartilage damage repair (Nettles et al., 2002).

During the process of repair, the gel will be gradually replaced by cartilage tissue. Generally speaking, the degradation time of materials should match the production speed of the new tissue. Rapid degradation of the scaffold affects the adhesion of chondrocytes, while slow degradation hinders cells' proliferation and matrix secretion (Bettinger, 2011; Kosuge et al., 2013). From H \& E staining and immunohistochemical staining results, we observed that chondrocytes cultured in hydrogel in vitro can grow and secrete extracellular matrix-collagen II. However, the residual scaffold still can be found in the defects before the defects are fully repaired by regenerative tissue. $H \&$ E staining showed that incomplete degradation of the scaffold was visible in the experimental group at the fourth week (Fig. 5g). The scaffold was gradually degraded and absorbed with time until there was no evidence of it at the eighth week in the experimental group (Fig. 5h). By contrast, the scaffold in the blank group was degraded slowly and was still in evidence after twelve weeks. That is why histological assessment in the blank group was lower than that in the control group (Fig. 4b). It is notable that the scaffold was degraded quicker in the experimental group than in the blank group. This may be attributed to the quicker formation of the new tissue in the experimental group, and more active metabolism has promoted the degradation of the scaffold.

The mechanical intensity of the scaffold was another factor that affected cartilage repair. In this research, the mechanical intensity of the regenerative articular cartilage was tested, but the results were not satisfactory (data not presented). The poor mechanical properties of the hydrogel scaffold were its main disadvantage. However, some scaffolds with poor mechanical properties still display excellent prospects in applications such as autologous chondrocyte implantation and matrix-induced autologous chondrocyte implantation (Redman et al., 2005; Willers et al., 2007; Hao et al., 2010). On the other hand, articular cartilage is composed of chondrocytes, collagen, proteoglycan, and water, and $80 \%$ of the fresh articular cartilage is water. Compared with other cartilage, articular cartilage has unique biological properties, such as permeability and viscoelasticity (Broom and Oloyede, 1998). Therefore, the structure and the physicochemical property of the articular cartilage are similar to those of hydrogel. As a result, it was suggested that chitosan hydrogel could be the suitable scaffold for constructing tissue-engineered articular cartilage.

\section{Conclusions}

The results suggest that preliminary tissueengineered cartilage has been successfully constructed in vitro. Chondrocytes on the hydrogel could secrete 
specific extracellular matrix. The tissue-engineered cartilage significantly improved the repair of damaged articular cartilage in terms of gross morphology and histology. We conclude that damaged cartilage can be repaired by tissue-engineered cartilage constructed with chitosan hydrogel prepared in our lab.

\section{Compliance with ethics guidelines}

Ming ZHAO, Zhu CHEN, Kang LIU, Yu-qing WAN, Xu-dong LI, Xu-wei LUO, Yi-guang BAI, Ze-long YANG, and Gang FENG declare that they have no conflict of interest.

All institutional and national guidelines for the care and use of laboratory animals were followed.

\section{References}

Bettinger, C.J., 2011. Biodegradable elastomers for tissue engineering and cell-biomaterial interactions. Macromol. Biosci., 11(4):467-482. [doi:10.1002/mabi.201000397]

Brittberg, M., Peterson, L., 1998. Introduction of an articular cartilage classification. ICRS Newsletter., 1:5-8.

Brittberg, M., Lindahl, A., Nilsson, A., et al., 1994. Treatment of deep cartilage defects in the knee with autologous chondrocyte transplantation. N. Engl. J. Med., 331(14): 889-895. [doi:10.1056/NEJM199410063311401]

Broom, N.D., Oloyede, A., 1998. The importance of physicochemical swelling in cartilage illustrated with a model hydrgel system. Biomaterials, 19(13):1179-1188. [doi:10. 1016/S0142-9612(98)00017-9]

Chen, Z., Zhao, M., Liu, K., et al., 2014. Novel chitosan hydrogel formed by ethylene glycol chitosan, 1,6-diisocyanatohexan and polyethylene glycol-400 for tissue engineering scaffold: in vitro and in vivo evaluation. J. Mater. Sci.: Mater. Med., 25(8):1903-1913. [doi:10.1007/s10856-014-5223-3]

de Franceschi, L., Grigolo, B., Roseti, L., et al., 2005. Transplantation of chondrocytes seeded on collagen-based scaffold in cartilage defects in rabbits. J. Biomed. Mater. Res. A, 75(3):612-622. [doi:10.1002/jbm.a.30471]

Dzioba, R.B., 1988. The classification and treatment of acute articular cartilage lesions. Arthroscopy, 4(2):72-80. [doi: 10.1016/S0749-8063(88)80067-7]

Fong, E.L., Watson, B.M., Kasper, F.K., et al., 2012. Building bridges: leveraging interdisciplinary collaborations in the development of biomaterials to meet clinical needs. $A d v$. Mater., 24(36):4995-5013. [doi:10.1002/adma.201201762]

Francis Suh, J.K., Matthew, H.W., 2000. Application of chitosan-based polysaccharide biomaterials in cartilage tissue engineering: a review. Biomaterials, 21(24): 2589-2598. [doi:10.1016/S0142-9612(00)00126-5]

Gentili, G., Cancedda, R., 2009. Cartilage and bone extracellular matrix. Curr. Pharm. Design, 15(12):1334-1348. [doi:10. 2174/138161209787846739]

Hao, T., Wen, N., Cao, J.K., et al., 2010. The support of matrix accumulation and the promotion of sheep articular cartilage defects repair in vivo by chitosan hydrogels. Osteoarthritis Cartilage, 18(2):257-265. [doi:10.1016/j.joca.
2009.08.007]

Holmes, J.S., 2004. Articular cartilage injuries in the athlete's knee: current concepts in diagnosis and treatment. South. Med. J., 97(8):742-747. [doi:10.1097/00007611-2004 08000-00010]

Iwasa, J., Engebretsen, L., Shima, Y., 2009. Clinical application of scaffolds for cartilage tissue engineering. Knee Surg. Sports Traumatol. Arthrosc., 17(6):561-577. [doi:10. 1007/s00167-008-0663-2]

Jin, R., Moreira Teixeira, L.S., Dijkstra, P.J., et al., 2009. Injectable chitosan-based hydrogels for cartilage tissue engineering. Biomaterials, 30(13):2544-2551. [doi:10. 1016/j.biomaterials.2009.01.020]

Kim, I.Y., Seo, S.J., Moon, H.S., et al., 2008. Chitosan and its derivatives for tissue engineering applications. Biotechnol. $A d v ., 26(1): 1-21$. [doi:10.1016/j.biotechadv.2007.07.009]

Kosuge, D., Khan, W.S., Haddad, B., et al., 2013. Biomaterials and scaffolds in bone and musculoskeletal engineering. Curr. Stem Cell Res. Ther., 8(3):185-191. [doi:10.2174/ 1574888X11308030002]

Kuo, Y.C., Lin, C.Y., 2006. Effect of genipin-crosslinked chitin-chitosan scaffolds with hydroxyapatite modifications on the cultivation of bovine knee chondrocytes. Biotechnol. Bioeng., 95(1):132-144. [doi:10.1002/bit.21007]

Lee, K.Y., Mooney, D.J., 2001. Hydrogels for tissue engineering. Chem. Rev., 101(7):1869-1879. [doi:10.1021/ cr000108x]

Liu, J., Wu, D.Y., Wang, T., 2010. Research progress in antibacterial activity of chitosan and development of antimicrobial textile. J. Textile Res., 31(7):146-150.

Mainil-Varlet, P., Aigner, T., Brittberg, M., et al., 2003. Histological assessment of cartilage repair: a report by the Histology Endpoint Committee of the International Cartilage Repair Society (ICRS). J. Bone Joint Surg. Am., 85-A(Suppl. 2):45-57.

Mallick, K.K., Cox, S.C., 2013. Biomaterial scaffolds for tissue engineering. Front. Biosci., 5:341-360. [doi:10.2741/ E620]

Messent, E.A., Ward, R.J., Tonkin, C.J., et al., 2007. Osteophytes, juxta-articular radiolucencies and cancellous bone changes in the proximal tibia of patients with knee osteoarthritis. Osteoarthritis Cartilage, 15(2):179-186. [doi:10. 1016/j.joca.2006.06.020]

Nettles, D.L., Elder, S.H., Gilbert, J.A., 2002. Potential use of chitosan as a cell scaffold material for cartilage tissue engineering. Tissue Eng., 8(6):1009-1016. [doi:10.1089/ 107632702320934100]

Ochi, M., Uchio, Y., Kawasaki, K., et al., 2002. Transplantation of cartilage-like tissue made by tissue engineering in the treatment of cartilage defect of the knee. J. Bone Joint Surg. Br., 84(4):571-578. [doi:10.1302/0301-620X.84B4. 11947]

Perka, C., Spitzer, R.S., Lindenhayn, K., et al., 2000. Matrix-mixed culture: new methodology for chondrocyte culture and preparation of cartilage transplants. J. Biomed. Mater. Res., 49(3):305-311. [doi:10.1002/(SICI)10974636(20000305)49:3<305::AID-JBM2>3.3.CO;2-0] 
Peterson, L., Minas, T., Brittberg, M., et al., 2000. Two- to 9-year outcome after autologous chondrocyte transplantation of the knee. Clin. Orthop. Relat. Res., 374:212-234. [doi:10.1097/00003086-200005000-00020]

Peterson, L., Vasiliadis, H.S., Brttiberg, M., et al., 2010. Autologous chondrycyte implantation a long-term follow up. Am. J. Sports Med., 38(6):1117-1124. [doi:10.1177/ 0363546509357915]

Qiao, P.Y., Li, F.F., Dong, L.M., et al., 2014. Delivering MC3T3-E1 cells into injectable calcium phosphate cement through alginate-chitosan microcapsules for bone tissue engineering. J. Zhejiang Univ.-Sci. B (Biomed. \& Biotechnol.), 15(4):382-392. [doi:10.1631/jzus.B1300132]

Redman, S.N., Oldfield, S.F., Archer, C.W., 2005. Current strategies for articular cartilage repair. Eur. Cell Mater., 9:23-32.

Richter, W., 2009. Mesenchymal stem cells and cartilage in situ regeneration. J. Intern. Med., 266(4):390-405. [doi: 10.1111/j.1365-2796.2009.02153.x]

Roach, H.I., Aigner, T., Soder, S., et al., 2007. Pathobiology of osteoarthritis: pathomechanisms and potential therapeutic targets. Curr. Drug Targets, 8(2):271-282. [doi:10.2174/ 138945007779940160]

Steadman, J.R., Rodkey, W.G., Briggs, K.K., 2002. Microfracture to treat full-thickness chondral defects: surgical technique, rehabilitation, and outcomes. J. Knee Surg., 15(3):170-176.

Wakitani, S., Goto, T., Pineda, S.J., et al., 1994. Mesenchymal cell-based repair of large, full-thickness defects of articular cartilage. J. Bone Joint Surg. Am., 76(4):579-592.

Wakitani, S., Goto, T., Young, R.G., et al., 1998. Repair of large full-thickness articular cartilage defects with allograft articular chondrocytes embedded in a collagen gel. Tissue Eng., 4(4):429-444. [doi:10.1089/ten.1998.4.429]

Willers, C., Partsalis, T., Zheng, M.H., 2007. Articular cartilage repair: procedures versus products. Expert Rev. Med. Devices, 4(3):373-392. [doi:10.1586/17434440.4.3.373]

\section{中文概要}

题 目: 新型壳聚糖水凝胶结合软骨细胞修复兔关节软骨 缺损的实验研究

目 的: 评估壳聚糖水凝胶结合肋软骨细胞构建的新 型组织工程软骨对兔关节软骨缺损的修复效 果。

创新点: 利用自主研发的具有良好生物相容性和稳定性的 壳聚糖水凝胶与软骨细胞, 在体外初步构建组织 工程软骨，并尝试利用其修复缺损的关节软骨， 从而为关节软骨缺损的修复提供了一种新的治疗 方法。

方 法: 取兔肋软骨体外培养扩增, 获得 P2 代软骨细胞, 将其种植到冻干的壳聚糖水凝胶上, 体外培养一 周, 获得初步构建的组织工程软骨。构建兔膝关 节软骨缺损模型, 并分为 3 组: 实验组植入 组织工程软骨; 对照组植入壳聚糖水凝胶; 空白组不做任何处理。分别于术后 $4 、 8$ 和 12 周取材，通过大体观察、苏木精-伊红染色、 番红-O 染色及 II 型胶原免疫组化染色等方法 观察缺损关节软骨的修复情况, 并用国际关 节软骨修复协会 (ICRS) 制定的评分法进行大 体及组织学评分。

结 论: 兔肋软骨细胞能在实验室自主构建的壳聚糖水凝 胶上增殖并分泌细胞外基质（图 2), 植入到兔关 节软骨缺损处后, 对缺损关节软骨具有良好的 修复作用 (图 3 和 5 ), 且修复是一种完全的 结构性的修复 (图 5 7)。

关键词: 关节软骨修复; 组织工程软骨; 壳聚糖水凝胶 